\title{
Plasticity in the gut microbial community and uptake of Enterobacteriaceae (Gammaproteobacteria) in Bombus terrestris bumblebees' nests when reared indoors and moved to an outdoor environment
}

\author{
Laurian Parmentier $^{1}$, Ivan Meeus ${ }^{1}$, Hadi MosallaneJad ${ }^{1}$, Dirk C. de GraAF ${ }^{2}$, \\ Guy SMAGGHE ${ }^{1}$
}

\footnotetext{
${ }^{1}$ Department of Crop Protection, Faculty of Bioscience Engineering, Ghent University, Coupure links 653, Ghent 9000, Belgium

${ }^{2}$ Laboratory of Molecular Entomology and Bee Pathology, Faculty of Sciences, Ghent University, Ghent 9000, Belgium
}

Received 7 April 2015 - Revised 8 August 2015 - Accepted 15 September 2015

\begin{abstract}
Bombus nests consisting of one queen, brood, and worker adults, are produced indoors for biological pollination in agriculture. In this study, we investigated the gut microbial community in workers of Bombus terrestris when the environment is stable (indoors) or variable (outdoors). When nests were reared indoors under standardized conditions, we identified a small gut microbial community consisting of Neisseriaceae, Orbaceae, Lactobacillaceae, and Bifidobacteriaceae, and the age of bumblebee nests and workers did not affect the alpha and beta diversity, confirming a stable microbiota. Secondly, when indoor-reared nests were moved to outdoors, we observed a major shift in the microbial community, especially in the newborn workers fully developed in the outdoor conditions, with a significant colonization of Enterobacteriaceae. Our new findings are discussed in relation to hostassociated core and non-core bacteria in bumblebees including possible implications for host functioning.
\end{abstract}

\section{Bombus terrestris / generalist pollinator / microbiota / plasticity / Enterobacteriaceae / reared bumblebees}

\section{INTRODUCTION}

Insects easily outnumber other animal clades in the world inhabiting a diverse range of ecosystems. Insects are, in many cases, dependent on functions provided by bacterial symbionts (Brownlie and Johnson 2009; Basset et al. 2012), including gut microbiota with beneficial functions in nutrition (Salem et al; 2012) and protection (Dillon and Charnley 1986, 1988). Some insects harbor gut

Electronic supplementary material The online version of this article (doi:10.1007/s13592-015-0393-7) contains supplementary material, which is available to authorized users.

Corresponding author: G. Smagghe, guy.smagghe@ugent.be

Manuscript editor: David Tarpy communities of specific bacteria, which is especially true in social insects (Engel and Moran 2013). Within the clade of social bees, research on microbial communities has been focusing on honeybees (Martinson et al. 2012; Moran et al. 2012) and bumblebees (Koch and Schmid-Hempel 2011b; Koch et al. 2012; Meeus et al. 2013; Li et al. 2015; Praet et al. 2015) because of their important pollinating role in natural ecosystems and of many agricultural crops, together with a growing evidence of their dramatic declines worldwide (Ghazoul 2005; Lebuhn et al. 2013).

The social nesting behavior of honeybees and bumblebees enables the transfer of gut bacteria over the generations, allowing a close association with the host (Powell et al. 2014). Indeed, social bees, as opposed to solitary bees, generally harbor host-associated bacterial groups, defined as "core 
set," and the latter are generally found in the gut microbiota of social bees (Kaltenpoth 2011; Koch and Schmid-Hempel 2011b; Cariveau et al. 2014). Core bacteria dominating the gut of social honeybees and bumblebees have been implicated in beneficial functions such as digestion and protection (Klungness and Peng 1984; Forsgren et al. 2010; Koch et al. 2012; Vásquez et al. 2012; Kwong et al. 2014). Multiple conditions have been reported influencing the bacterial gut community, including the environment, host age, habitat, pathogens, and diet (Dillon et al. 2010; Evans and Schwarz 2011; Hu et al. 2014; Yun et al. 2014).

Currently, the buff-tailed bumblebee Bombus terrestris is reared indoors for the biological pollination in agriculture. This species is widely distributed and native to Europe, coastal North Africa, and West and Central Asia (Goulson 2010). Due to the decline of bees worldwide, research has been focused on diseases (Fürst et al. 2014) and pathogen spillover (Meeus et al. 2011). Besides, there has been little research on the gut microbial community and the dynamics of indoor-reared bumblebees foraging in an environment outdoors.

In this paper, we used $16 \mathrm{~S}$ rRNA Illumina deep sequencing to identify the gut microbial community in workers of $B$. terrestris from indoor-reared nests. In a first experiment, we determined the growth and richness of this microbial community when the nests were kept under indoor conditions. Our ambitions were to determine the dominant bacteria in indoor-reared nests and the stability of these bacteria in function of the age of the adults and the nest. In a second experiment, the indoorreared bumblebee nests were moved to outdoors to investigate the plasticity of the gut microbial community in the workers of different ages. In summary, the new data found in this study are of use to discuss the relationship between core and non-core bacteria in bumblebees and to generate new insights in the gut microbial stability of indoorreared bumblebee hives when moved to outdoors.

\section{MATERIAL AND METHODS}

\subsection{Bumblebee nests and insect labeling}

Six nests of $B$. terrestris were obtained from a bumblebee mass-rearing facility (Biobest,
Westerlo, Belgium). All colonies contained one queen, new queen brood but without adult workers. The nests were kept in the lab at standard conditions of $28-30{ }^{\circ} \mathrm{C}$ and $60-65 \%$ relative humidity and continuous darkness, and they were fed ad libitum with artificial nectar (Biogluc ${ }^{\circledR}$, Biobest) and pollen clumps (Soc. Coop. Apihurdes, Pinofranqueado-Caceres, Spain) as energy and protein sources, respectively (Mommaerts et al. 2006). For the six bumblebee nests, we labeled the queen and then also each newborn worker adult on a daily basis with a unique marking disc (Bijenhof, Bissegem, Belgium) to the dorsal thorax. The six nests were used for two experiments where in the first experiment, three nests were kept indoors under the standard conditions as described earlier, while in the second experiment, the other three nests were moved outdoors. The experiments were carried out between end August up to end September and early October 2012.

\subsection{Experiment 1: bumblebee nests kept under standard conditions indoors}

Three nests were followed for 60 days during the rearing period in the lab under standard conditions. We aimed to investigate the impact of age of the nest and the individual bumblebee worker in the nest. Therefore, we defined two age periods of the nest: young nest ( $\mathrm{Y}^{\mathrm{n}}$; 4- to 11-day old nest) and old nest $\left(\mathrm{O}^{\mathrm{n}} ; 40\right.$ - to 46-day old nest) and two age periods of the workers: young bee $\left(\mathrm{Y}^{\mathrm{b}} ; 4\right.$ to 10 days old) and old bee $\left(\mathrm{O}^{\mathrm{b}} ; 28\right.$ to 35 days old). When combining the age period of the nest with the age of the individual workers, we defined three test groups: young nest, young bee $\left(\mathrm{Y}^{\mathrm{n}} \mathrm{Y}^{\mathrm{b}}\right)$; old nest, old bee $\left(\mathrm{O}^{\mathrm{n}} \mathrm{O}^{\mathrm{b}}\right)$; and old nest, young bee $\left(\mathrm{O}^{\mathrm{n}} \mathrm{Y}^{\mathrm{b}}\right)$. In total, we sampled eight workers per test group. Sampled bumblebees were stored individually at $-70{ }^{\circ} \mathrm{C}$ until dissection of the gut.

\subsection{Experiment 2: indoor-reared bumblebee nests moved to an outdoor environment}

In parallel to experiment 1 , we kept the three other nests indoors in the lab so that they developed an initial indoor-reared microbiota. When 
placed outdoors, the three nests developed further under natural conditions during 50 days. The outdoors study site $\left(51^{\circ} 0132.57 \mathrm{~N} ; 3^{\circ} 4246.10\right.$ $\mathrm{O})$ was located in a residential area in the city of Ghent (Belgium) with landscape metrics of an urbanized environment (Parmentier et al. 2014). As shown in Figure S1, each nest was placed in a separate polystyrene-box $(25 \times 35 \times 25 \mathrm{~cm})$ with the entrance directed to the east and further treated as described in Parmentier et al. (2014).

In the outdoors experiment, we defined three age periods of the nests: young nest $\left(\mathrm{Y}^{\mathrm{n}}, 4\right.$ - to $11-$ day old nest); middle-aged nest $\left(\mathrm{M}^{\mathrm{n}}, 28\right.$ - to 34day old nest); and old nest $\left(\mathrm{O}^{\mathrm{n}}, 40\right.$ - to 46-day old nest), and three age periods of the workers: young bumblebee ( $\mathrm{Y}^{\mathrm{b}}, 4$ to 10 days old); middle-aged bumblebee ( $\mathrm{M}^{\mathrm{b}}$, 18 to 23 days old); and old bumblebee $\left(\mathrm{O}^{\mathrm{b}}, 28\right.$ to 35 days old). Here, we defined "young" and "old" ages identical as in experiment 1 , and the extra "middle" age was introduced in order to sample sufficient forager and nesting bumblebees. We defined a "forager" and "nesting" bumblebee as a worker typically showing a nesting and foraging function, respectively. Thus, in total, we defined five test groups: young nest, young bumblebee $\left(\mathrm{Y}^{\mathrm{n}} \mathrm{Y}^{\mathrm{b}}\right)$; old nest, old bumblebee $\left(\mathrm{O}^{\mathrm{n}} \mathrm{O}^{\mathrm{b}}\right)$; old nest, young bumblebee $\left(\mathrm{O}^{\mathrm{n}} \mathrm{Y}^{\mathrm{b}}\right)$; middle-aged nest, young bumblebee $\left(\mathrm{M}^{\mathrm{n}} \mathrm{Y}^{\mathrm{b}}\right)$; and middle-aged nest, middle-aged bumblebee $\left(\mathrm{M}^{\mathrm{n}} \mathrm{M}^{\mathrm{b}}\right)$. Following our definition, at nest level, initial sampling in the young nests $\mathrm{Y}^{\mathrm{n}} \mathrm{Y}^{\mathrm{b}}$ was achieved under indoor conditions (same as indoor-reared hives in experiment 1), whereas sampling in all other test groups was achieved outdoors. In total, we sampled $7 \pm 1$ nesting bees per test group $\mathrm{Y}^{\mathrm{n}} \mathrm{Y}^{\mathrm{b}}, \mathrm{O}^{\mathrm{n}} \mathrm{O}^{\mathrm{b}}$, and $\mathrm{O}^{\mathrm{n}} \mathrm{Y}^{\mathrm{b}}$. In the test groups $M^{n} Y^{b}$ and $M^{n} M^{b}, 6 \pm 2$ nesting and $6 \pm 2$ foraging workers were sampled per test group, respectively. Sampled bumblebees were stored individually at $-70{ }^{\circ} \mathrm{C}$ until dissection of the gut.

To follow and record individually foraging workers leaving or entering the nest, we placed a webcam (Logitech ${ }^{\circledR}$ C920 HD Pro, Lausanne, Switzerland) with a top view on the entrance of each nest and optimized in "move-detection" function (Online Resource Figure S1). The flight duration of individual foragers was calculated and we ranked them daily based on the numbers of flights under good weather conditions. We selected foraging workers (foragers) that had at least five flights per day under good weather conditions (excluding rainy days and temperatures $<15^{\circ} \mathrm{C}$ ).

\subsection{Gut samples processing, Illumina sequencing, and identification}

The gut, including the crop, was dissected from the different bumblebee workers and transferred individually into a 1.5-mL Eppendorf tube. All equipment used was disinfected with $75 \%$ ethanol between each individual dissection. The extraction of bacterial DNA was done as previously described (Meeus et al. 2013), and then the V4 region of the ribosomal RNA (rRNA) was amplified in triplicate, using the $515 \mathrm{~F}$ and $806 \mathrm{R}$ primers elongated with multiplex identifiers (Caporaso et al. 2011). The composite primers include the $16 \mathrm{~S}$ primer sites, a different nucleotide barcode (on the 806R primer) and the Illumina adapter sequences necessary for bridge amplification on the Illumina MiSeq flow cell (Illumina, San Diego, CA). Amplicons were normalized, mixed at equimolar concentrations, purified, and further concentrated as previously described (Meeus et al. 2015 ) to produce a 8 -pM sequencing library. To increase sequence diversity, $20 \%$ denaturated Illumina PhiX Control V3 library was admixed. Cluster generation and $2 \times 150$ paired-end sequencing was performed on one Illumina Miseq flowcell using an Illumina Miseq Reagent Kit v.2. Custom sequencing primers were added in a final concentration of $0.5 \mu \mathrm{M}$ (Meeus et al. 2015). Base calling and primary quality assessments and demultiplexing were performed at the Miseq sequencing platform of Laboratory for Pharmaceutical Biotechnology (UGent) using Illumina's Basespace genomics cloud computing environment.

The pooled data set contained 3,353,558 double read sequences. A unique barcode was used to separate each sequence to its sample of origin. Sequences were analyzed with the Mothur software v.1.31.1 following the standard procedure (Schloss et al. 2009). In brief, the complexity was reduced by a training set of base pairs before clustering into operational taxonomic units (OTUs). The procedure includes denoising of 
unique sequences (preclustering all sequences with 1 mutation on $100 \mathrm{bp}$ ) and removing of chimeras using the UCHIME algorithm (Edgar et al. 2011). The distance matrix was calculated and clustering with a 0.03 cutoff level resulted in 461 OTUs. To exclude sequencing errors, we only kept these OTUs that had more than $0.5 \%$ of the sequence reads in every sample. This procedure resulted in 20 OTUs, representing $99 \%$ of the total reads.

\subsection{Classification and verification of new OTUs}

The taxonomic identity of each OTU was achieved by alignment of each sequence with the Bacterial SILVA SEED database (training set), supplemented with host-specific sequences (i.e., host Bombus or Apis) to improve classification (Newton and Roeselers 2012). The identity of each bacterial OTU was achieved by a two-way strategy. First, BLASTn searches of the representative sequences were achieved. Second, when a $100 \%$ base pair alignment was not obtained with representative sequences of Bombus hosts of a previous dataset (Meeus et al. 2015) (available at Genbank KM03050545 to KM03050553), a semi-nested PCR with a universal Eub8F or 984yR primer combined with an OTU-specific primer was applied. All new sequences were deposited at Genbank (KP410382 to KP410393) and raw Illumina data reads of all samples were submitted to SRA database of Genbank under project ID PRJNA270053 (Tables I, II).

In our study, we used a classification system of bacterial OTUs to separate core and non-core OTUs (Koch and Schmid-Hempel 2011a; Koch et al. 2013; Cariveau et al. 2014). We defined Neisseriaceae, Orbaceae, Lactobacillaceae, and Bifidobacteriaceae as core families for bumblebees in accordance with Kwong et al. (2014) and Meeus et al. (2015). All other bacteria are regarded as non-core OTUs.

\subsection{Statistical analysis}

Samples were normalized to the smallest number of reads for a given sample $(n=49,420)$ using the normalize.shared function (Schloss et al.
2009) and then used to generate the diversity calculators. Calculators "Sobs" (observed number of species), "Chao1" and the Shannon index were used to map the alpha diversity (Schloss et al. 2009). Differences in alpha diversity between pre-defined test groups of indoor-reared and outdoor-developed nests were determined by the non-parametric Mann-Whitney $U$ test (SPSS vs. 21). Significance cutoff values were set at $95 \%$ $(\alpha=0.05)$. Differences in similarity between test groups (even sampling) were calculated by analysis of similarities (ANOSIM). To visualize differences in the bacterial community (beta diversity), non-metric multidimensional scaling (NMDS) was used based on a Bray-Curtis similarity matrix of the square root transformed normalized relative abundance of the different OTUs per sample (Primer 6 version 6.1.10). An in-depth multivariate analysis was performed in order to evaluate dispersion effects between test groups by using the R package "mvabund" selecting the "anova.manyglm" call. After checking model assumptions by plotting "meanvar" and "manyglm" matrixes, data was further processed as a "negative binomial" distribution, a typical option for abundance count data in the mvabund-package (Wang et al. 2012).

\section{RESULTS}

\subsection{Workers of indoor-reared nests harbored a small microbial gut community of stable composition}

In total, we sampled 24 nesting bumblebee workers from three indoor-reared nests, having representative samples of bumblebees in three age groups $\left(\mathrm{Y}^{\mathrm{n}} \mathrm{Y}^{\mathrm{b}}, \mathrm{O}^{\mathrm{n}} \mathrm{Y}^{\mathrm{b}}, \mathrm{O}^{\mathrm{n}} \mathrm{O}^{\mathrm{b}}\right)$. Rarefaction curves (Figure $\mathrm{S} 2$ in the supplemental material) illustrate that an asymptote was achieved for 49,000 sequence read per sample and seven samples per test group, indicating sufficient sequencing depth. After OTU picking procedure, the microbial community of the indoor-reared workers (experiment 1 and initial indoor-reared bumblebees in experiment 2) clustered in 13 OTUs. Figure 1a shows the relative abundance of each OTU per test group. Most of the sequences are classified under known core OTUs (Table I), i.e., 


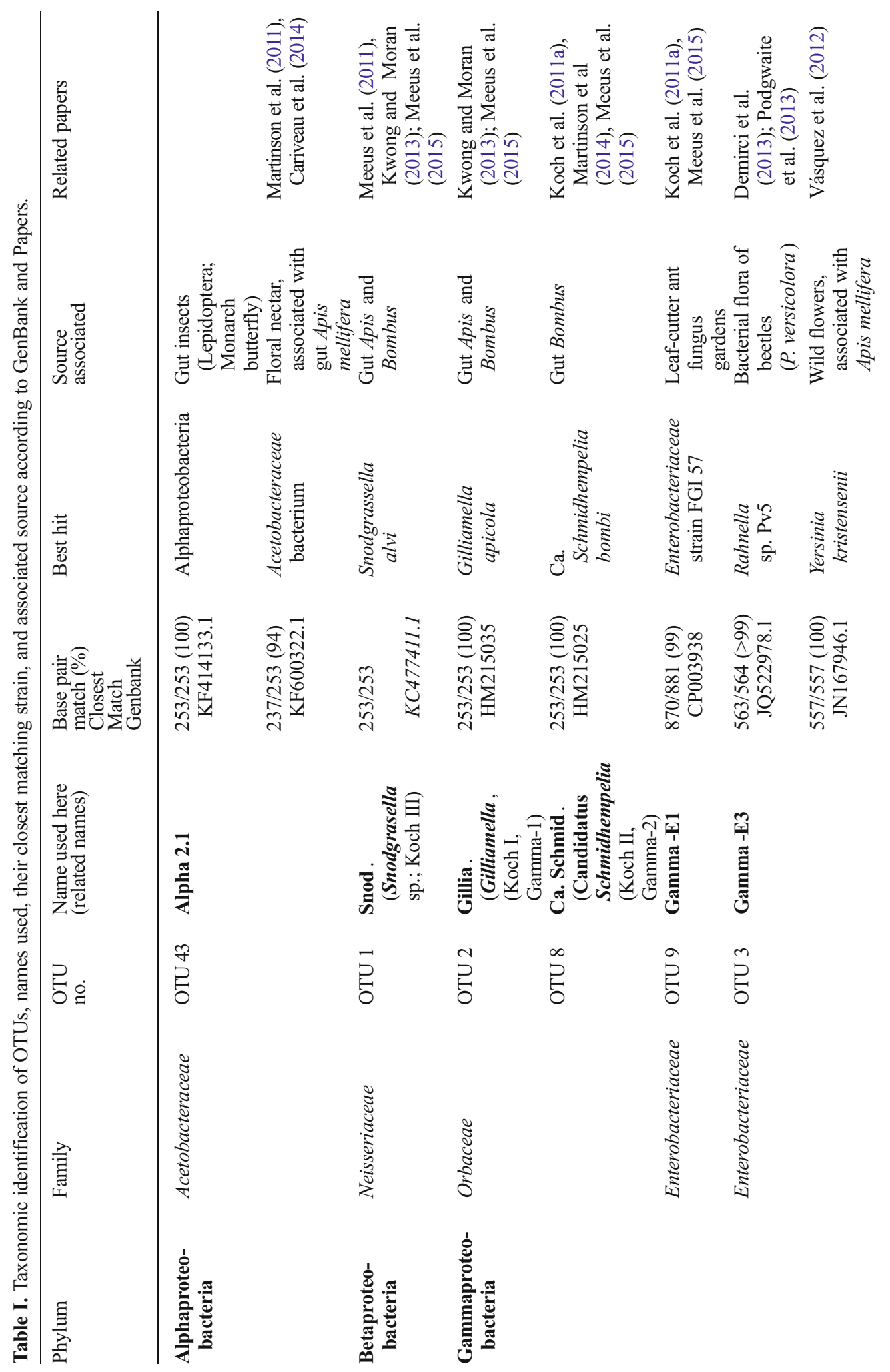




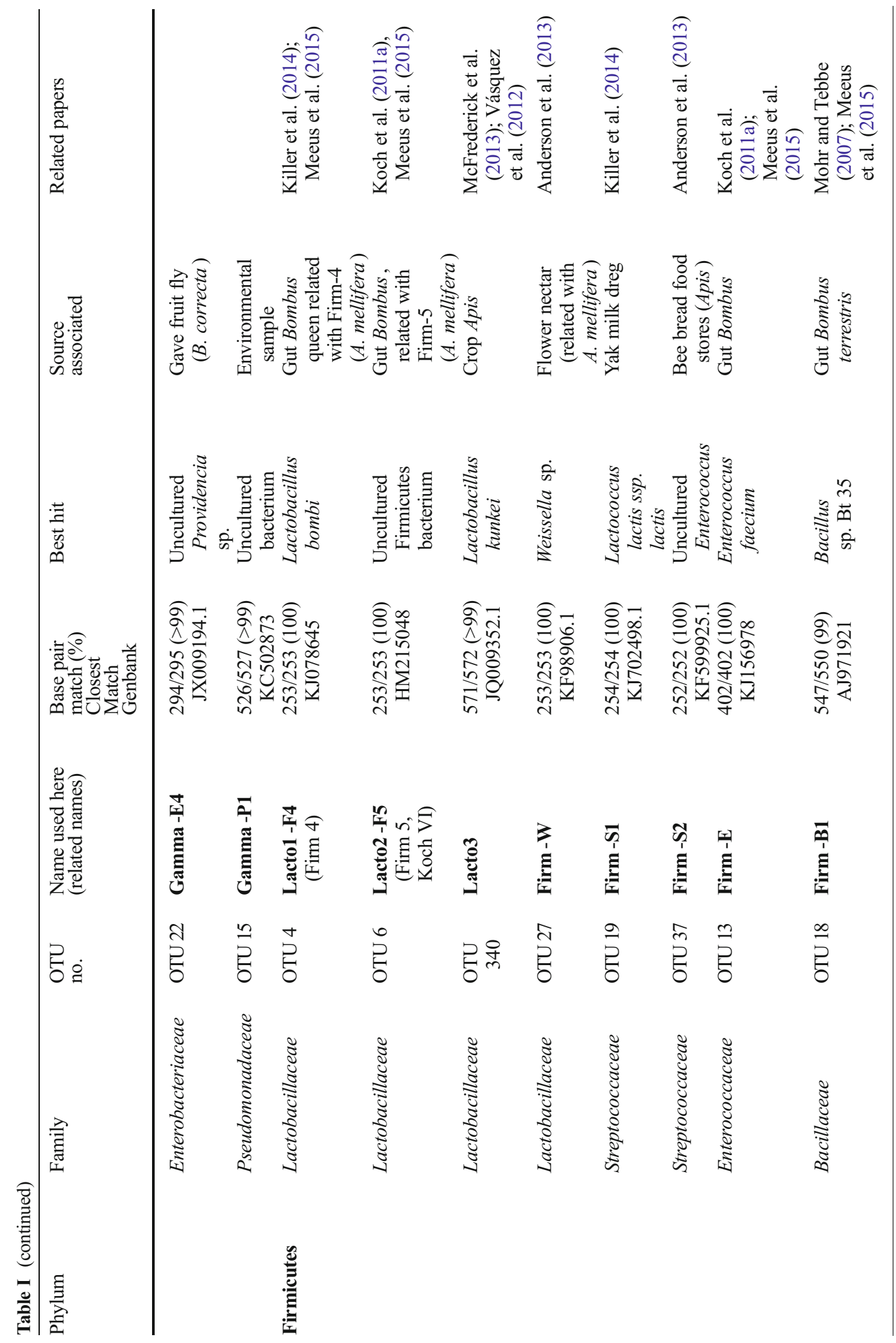




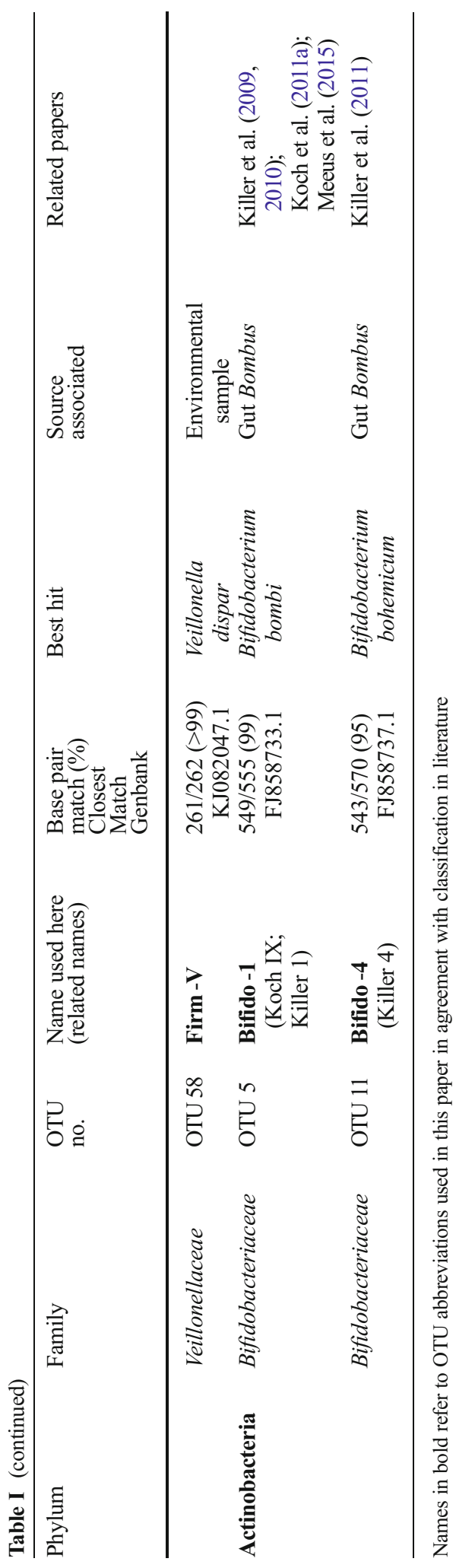

Snodgrasella (Snod.) classified under Neisseriaceae, Gilliamella (Gillia.) and Candidatus Schmidhempelia (Ca. Schmid.) belonging to Orbaceae, Lacto1-Firm4, Lacto2Firm5, Firm-B1, Firm-E, Firm-W, which are all Firmicutes, and Lacto3 classified under Lactobacillaceae, and Bifido-1 belonging to the Bifidobacteriaceae. A very small number of the reads belonging to Gamma-E1 and Gamma-E4 (both $<0.5 \%$ ), was classified under Enterobacteriaceae. When looking at alpha diversity calculators such as community richness (Sobs and Chao1) and community diversity (Shannon index) (Fig. 1b), no differences were observed between the test groups in the indoor-reared nests (MannWhitney $U$ test; $P$ values are given in Table III). Besides, looking at the 2-dimensional NMDS plots $\left(\mathrm{STRESS}_{1}=0.16\right)$ which is an indicator for beta diversity (Fig. 1c), no differences between test groups (ANOSIM, $R=0.11, P=0.51$ ) were observed, which was also confirmed by multiple univariate test statistics (all $P$ values $>0.05$, Table III). However, an in-depth multivariate analysis (MANOVA results using "mvabund" package, Table IV) estimated that there exist small differences $(\mathrm{Dev}=22.4, P=0.045)$ between the groups, indicating small dispersion effects between young and old bumblebee workers.

\subsection{Newborn workers showed a gut microbiota shift with a major colonization of Enterobacteriaceae when the nests were moved to outdoors}

In the second experiment, indoor-reared nests were moved to outdoors, and we sampled again 24 nesting bumblebee workers for the three test groups with sufficient depth (Figure S3). The microbial community in the initial indoor-reared workers $\mathrm{Y}^{\mathrm{n}} \mathrm{Y}^{\mathrm{b}}$ developed a typical microbiota as seen in experiment 1 (Figs. 1a and 2a) but workers from the nests moved to outdoors shifted completely after 6 weeks and new OTUs were taken up (Fig. 2a). Data analysis revealed 20 bacterial OTUs as summarized in Table I. Looking to alpha diversity (Fig. 2b), we detected no differences in community richness (Sobs: $P=0.306$ and Chaol: $P=0.507$ ), but a significant difference in community diversity (Shannon: 
Table II. OTU-specific primers combined with a universal 16S rDNA primer (Eub8F or 984yR).

\begin{tabular}{lll}
\hline Target & Primer & \\
\cline { 2 - 3 } & Forward & Reverse \\
\hline Alpha-2.1 & Eub8F AGAGTTTGATCMT & AGTGTGAGAGAGGATTGT \\
Gamma-E1 & GGCTCAG TGTCAAGT & GG 984yR GTAAGGTTCYTCGCGT \\
& CGGATGTAAAT & \\
& Eub8F AGAGTTTGATCMT & TCACATCCGACTTGACAGAC \\
Gamma-E3 & CGGTTTGTTAAGTCAGATGT & 984yR GTAAGGTTCYTCGCGT \\
& Eub8F AGAGTTTGATCMTGGCTCAG & ATGCAGTTCCCAAGTTAAGC \\
Gamma-E4 & GAATGGCATCTAAAACTGGT & GAATGGCATCTAAAACTGGT \\
Bifido-1 & Eub8F AGAGTTTGATCMTGGCTCAG & CAGTCTCCCCTACTGCACTC \\
Bifido-4 & GAGTGCAGTAGGGGAGACTG & 984yR GTAAGGTTCYTCGCGT \\
& Eub8F AGAGTTTGATCMTGGCTCAG & CAGTCTCCCCTCTGCACTC \\
Lacto3 & Eub8F AGAGTTTGATCMTGGCTCAG & CTGTCCTCTTCTGCACTCAA \\
Firm-W & Eub8F AGAGTTTGATCMTGGCTCAG & AGACGGTTATTTAAGTCCGA \\
Firm-S1 & GTGCATTGGAAACTGTTAGA & GTGCATTGGAAACTGTTAGA \\
& TTAACTGTGGAAGTGCTTTG & 984yR GTAAGGTTCYTCGCGT \\
Firm-S2 & GTGCATTGGAAACTGTTAGA & 984yR GTAAGGTTCYTCGCGT \\
Firm-V & GAGTATCGGAGAGGAAAGTG & 984yR GTAAGGTTCYTCGCGT
\end{tabular}

$P=0.002)$ in the group of young workers born in the old nest $\left(\mathrm{O}^{\mathrm{n}} \mathrm{Y}^{\mathrm{b}}\right)$. Indeed, the evenness of the gut microbiota is different in these young bumblebees due to the major uptake of new OTUs, especially Gamma-E2. Multivariate statistics (anova.manyglm) demonstrated a clear dispersion effect between the test groups ( $\mathrm{Dev}=585,665$, $P=0.001$ ). For the beta diversity (Fig. 2c), as represented in the NMDS plot (STRESS $=0.04$ ), we saw a clear separate cluster (ANOSIM, $R=0.99, P=0.001$ ), grouping all workers that were newborn in the old nest $\left(\mathrm{O}^{\mathrm{n}} \mathrm{Y}^{\mathrm{b}}\right)$.

When looking to differences between foraging and nesting workers from the nest moved to outdoors, we sampled 14 bumblebees with an equal number of foraging and nesting workers (test groups $M^{n} Y^{b}$ and $M^{n} M^{b}$ ) with sufficient depth (Figure S4 in supplementary material). Although we observed a significant difference between the age categories $\mathrm{M}^{\mathrm{n}} \mathrm{Y}^{\mathrm{b}}$ and $\mathrm{M}^{\mathrm{n}} \mathrm{M}^{\mathrm{b}}$ (anova.manyglm; Dev=204,923; $P=0.023$ ), both univariate and multivariate statistics calculated no significant differences between foraging and nesting workers or between nests of both ages (univariate: all $P$ values $>0.05$, multivariate: anova.manyglm; $\mathrm{Dev}=89,929, P=0.289$; see Table $\mathrm{S} 1$ in the supplementary material).

Finally, when comparing indoor-reared nests with nests that were moved to outdoors, we observed 11 additional OTUs in the gut microbiota, here identified as Alphaproteobacteria (Alpha-2.1), Enterobacteriaceae (Gamma-E3, Gamma-E4, Gamma-P1), Lactobacillaceae (Lacto-3, Firm-S1, Firm-S2, Firm-E, Firm-B1, Firm-V), and Bifidobacteriaceae (Bifido-4). Especially, one OTU had become very dominant, namely Gamma-E3 with a BLASTn match that is close to the environmental bacteria Rahnella sp. (Genbank JQ522978.1, >99 \% identity) and Yersinia kristensenii (Genbank JN167946.1, $100 \%$ identity) (Table I). Remarkably, we observed a dominance of Enterobacteriaceae in all shifted profiles. Indeed, considering all age groups in the old nests outdoors, NMDS visualization showed a cluster of all young bumblebees $\left(\mathrm{O}^{\mathrm{n}} \mathrm{Y}^{\mathrm{b}}\right)$ together with one old bumblebee $\left(\mathrm{O}^{\mathrm{n}} \mathrm{O}^{\mathrm{b}}\right)$ and two bumblebees of middle age $\left(\mathrm{O}^{\mathrm{n}} \mathrm{M}^{\mathrm{b}}\right)$ that all showed 
a

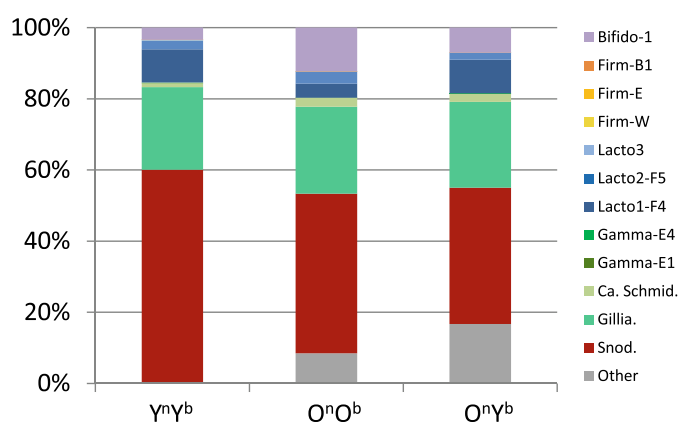

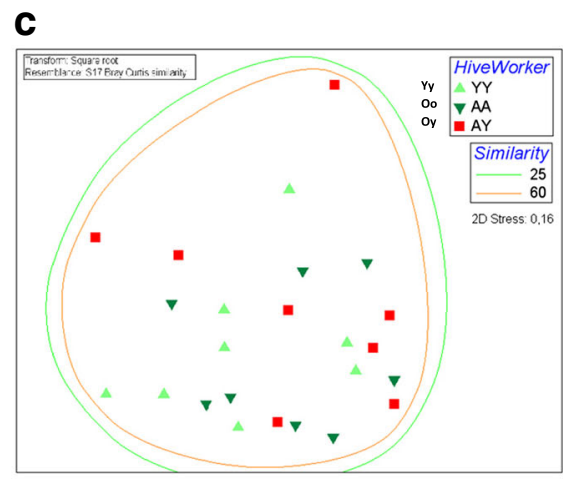

\section{b}

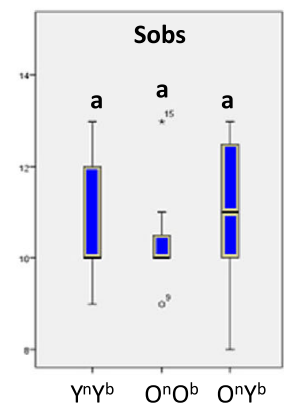

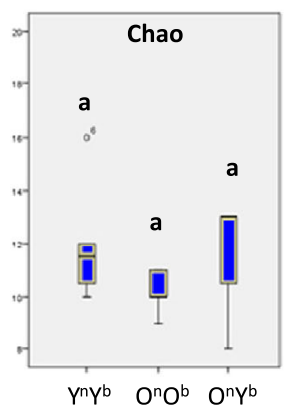

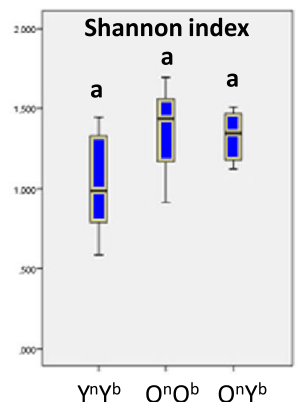

Figure 1. a Relative abundance of all OTUs comparing groups $\mathrm{Y}^{\mathrm{n}} \mathrm{Y}^{\mathrm{b}}, \mathrm{O}^{\mathrm{n}} \mathrm{O}^{\mathrm{b}}$, and $\mathrm{O}^{\mathrm{n}} \mathrm{Y}^{\mathrm{b}}$ of the indoor-reared $B$. terrestris nests. b Alpha diversity box plots (Sobs, Chao1, and Shannon index). c NMDS plots. $P=0.05$; MannWhitney $U$.

shifted profiles (ANOSIM, $R=0.99, P=0.005$, data not shown). Moreover, a relative dominance of Enterobacteriaceae correlated with a drop in the relative abundance of the core OTUs, as was clear in the individual profiles of all age groups (Figure S5).

\section{DISCUSSION}

In this study, we used Illumina deep sequencing technology of the 16S rRNA gene to investigate the gut microbial communities of bumblebee workers in nests reared indoors under controlled conditions in the lab in comparison to nests moved to outdoors after an initial indoor rearing period. We demonstrated that, in indoor-reared nest, the gut microbial community is stable over 6 weeks and all age groups are composed of a small number of identical core bacterial taxa (OTUs) classified under Neisseriaceae, Orbaceae, Lactobacillaceae, and Bifidobacteriaceae which can be grouped under core bacteria in bumblebees (Table I). This

Table III. $P$ values (Mann-Whitney $U$ test, $a=0.05$ ) of alpha diversity indicators Sobs, Chao, and Shannon index between test groups $\mathrm{Y}^{\mathrm{n}} \mathrm{Y}^{\mathrm{b}}, \mathrm{O}^{\mathrm{n}} \mathrm{Y}^{\mathrm{b}}$, and $\mathrm{O}^{\mathrm{n}} \mathrm{O}^{\mathrm{b}}$ for lab-reared hives.

\begin{tabular}{|c|c|c|c|c|c|c|}
\hline & \multicolumn{2}{|l|}{ Sobs } & \multicolumn{2}{|l|}{ Chao } & \multicolumn{2}{|c|}{ Shannon index } \\
\hline & $Y^{n} Y^{b}$ & $\mathrm{O}^{\mathrm{n}} \mathrm{O}^{\mathrm{b}}$ & $\mathrm{Y}^{\mathrm{n}} \mathrm{Y}^{\mathrm{b}}$ & $\mathrm{O}^{\mathrm{n}} \mathrm{O}^{\mathrm{b}}$ & $Y^{n} Y^{b}$ & $\mathrm{O}^{\mathrm{n}} \mathrm{O}^{\mathrm{b}}$ \\
\hline $\mathrm{O}^{\mathrm{n}} \mathrm{O}^{\mathrm{b}}$ & 0.382 & - & 0.051 & - & 0.442 & - \\
\hline $\mathrm{O}^{\mathrm{n}} \mathrm{Y}^{\mathrm{b}}$ & 0.382 & 0.105 & 0.505 & 0.065 & 0.234 & 0.999 \\
\hline
\end{tabular}


Table IV. $P$ values and Wald test scores of gut microbiota OTUs, multivariate and univariate comparison between test groups $\mathrm{Y}^{\mathrm{c}} \mathrm{Y}^{\mathrm{b}}, \mathrm{O}^{\mathrm{c}} \mathrm{O}^{\mathrm{b}}$, and $\mathrm{O}^{\mathrm{c}} \mathrm{Y}^{\mathrm{b}}$ of indoorreared hives $(P>0.05$; mvabund package. anova.manyglm test).

\begin{tabular}{lll}
\hline $\begin{array}{l}\text { Multivariate test } \\
\text { Df. }\end{array}$ & $\begin{array}{l}\text { Dev. } \\
21\end{array}$ & 22.632 \\
Univariate test & Walue \\
Bèta & 6.740 & 0.045 \\
Gamma-1 & 0.077 & $P$ value \\
Gamma-2 & 0.152 & 0.116 \\
Gamma-E2 & 2.344 & 0.981 \\
Lacto1-F4 & 1.224 & 0.981 \\
Lacto2-F5 & 0.251 & 0.726 \\
Lacto3 & 2.344 & 0.751 \\
Bifido-1 & 2.770 & 0.981 \\
Other & 6.539 & 0.726 \\
\hline
\end{tabular}

observation is in agreement with Meeus et al. (2015) who found that reared $B$. terrestris nests showed a subset of core bacteria compared to wild bumblebees. Therefore, our in-depth analysis confirms a homeostasis of OTUs and negligible inter- or intranest variability when bumblebees are fed pollen and artificial nectar ad libitum and then kept under constant conditions. A homeostasis in gut microbiota of artificial well-fed bumblebees has already been reported, even when bumblebees were primed with diseases (Koch and Schmid-Hempel 2012). Therefore, our results indicate that age of the nest and worker is of minor importance when assessing the core microbiota in the gut of bumblebees under controlled conditions.

Second, we investigated in-depth the gut microbiota of indoor-reared nests moved to an outdoors environment and observed that the alpha diversity calculators showed no significant differences, whereas the beta diversity calculators differed significantly compared to indoor-reared nests. Here, we discuss

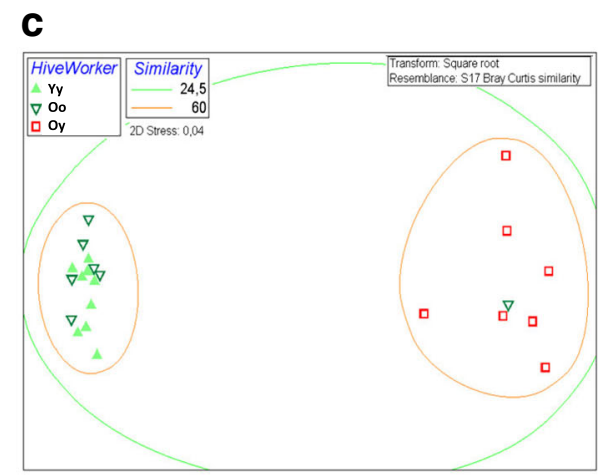

b
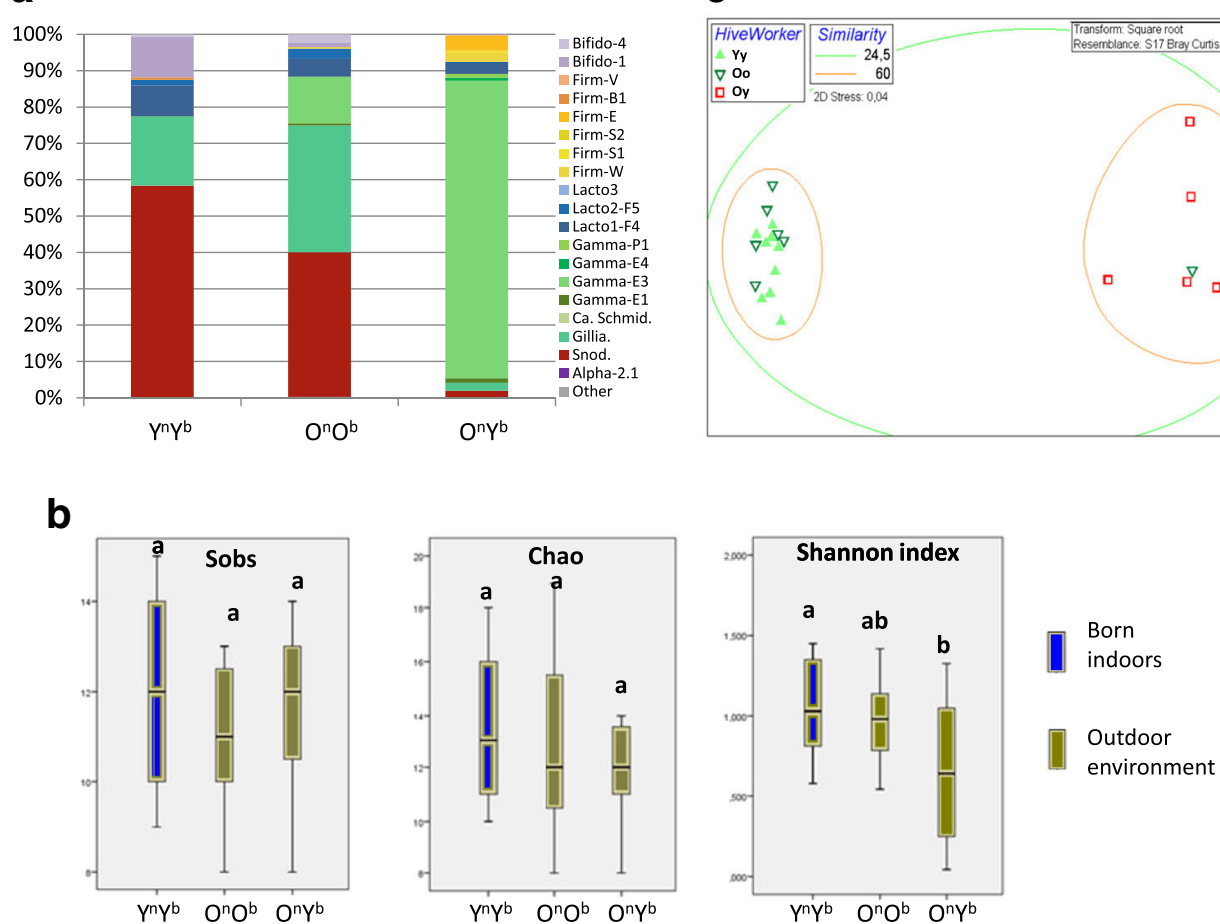

Figure 2. a Relative abundance of all OTUs comparing test groups $\mathrm{Y}^{\mathrm{n}} \mathrm{Y}^{\mathrm{b}}, \mathrm{O}^{\mathrm{n}} \mathrm{O}^{\mathrm{b}}$, and $\mathrm{O}^{\mathrm{n}} \mathrm{Y}^{\mathrm{b}}$ of indoor-reared B. terrestris nests moved to an outdoors environment. b Alpha diversity box plots (Sobs, Chao1, and Shannon index). c NMDS plots. $P=0.05$; Mann-Whitney $U$. 
our results with a study on social and herbivorous (pollen-feeding) Cephalotes varians ants (Hu et al. 2014). In this study, a comparison between laboratory-reared and field-caught workers revealed significant differences in the beta diversity of the gut microbiota. However, it should be remarked that, although the alpha diversity did not change between the bumblebee nests that were moved to outdoors and those that were kept indoors in our experiments, the outdoor-developed bumblebees showed extra OTUs, i.e., Firm-B1, Firm-E, Firm-W, Lacto3, Gamma-E1, and Gamma-E4. Therefore, a possible increase in alpha diversity outdoors could be hidden because the extra OTUs are small and dispersed, as was also the case in the $C$. varians study (Hu et al. 2014).

In this study, we observed that the profiles can change, mainly due to the colonization of Gamma-E3. A first hypothesis might be that the new bacteria were already present in low numbers in the nest of indoor-reared bumblebees, but that they were suppressed due to constant rearing conditions. Indeed, the outside environment itself with different nectar and pollen sources and other less optimal conditions is very different from the constant rearing conditions indoors promoting a small and stable microbiota (Meeus et al. 2015). In agreement, a shift in the gut bacterial diversity has been demonstrated in starving desert locusts Schistocerca gregaria compared to normal fed individuals (Dillon et al. 2010). Although, a second hypothesis remains possible as our results indicated that most of the new OTUs, including Gamma-E3, have been linked with environmental sources (BLASTn results shown in Table I). Thus, the colonization of Gamma-E3 can also be caused by the uptake of natural bacteria from the environment, probably by foraging workers.

Because the foraging workers had a more direct contact with the new outdoor environment compared to nesting workers, we expected that the former would have a microbiota that shifted earlier compared to the latter. However, in this study, we showed that the microbial community in the foraging workers did not shift earlier than the nesting workers of the same age but that the shift was mainly observed in the new workers (indifferent of their function) born in the old nest. We speculate that, when the workers had an established microbiota typically present in the young or middle-aged nest, they were less susceptible to take up new microbiota. Nonetheless, we believe that the foraging bumblebees can still bring the new bacteria from the outdoor environment into the nest, and this in turn resulted in a new inoculation in the old nest, that produced the newest generation of bumblebees.

Importantly, we observed that shifted profiles showed a high abundance of Enterobacteriaceae at the expense of the core bacteria Snodgrassella sp. and Gilliamella sp. (Fig. 2a). We showed that the Enterobacteriaceae bacteria are not exotic and most of the OTUs correspond with Genbank entries linked with hosts as Apis, Bombus, and other insects (Table I) (Anderson et al. 2013; Engel et al. 2013; Killer et al. 2011). We believe this is an important observation because Enterobacteriaceae are not regarded as core gut microbiota in social bees. However, it should be remarked that both Cariveau et al. (2014) and Meeus et al. (2015) also reported that the relative abundance of Enterobacteriaceae can be dominant (relative abundance up to $90 \%$ ) in some wild $B$. bimaculatus bumblebees and wild collected B. terrestris, respectively. Nevertheless, an Enterobacteriaceae-dominated gut microbial community can have its influence on the fitness of the bumblebee host or its resilience against pathogens (Cariveau et al. 2014). Indeed, a disruption in the homeostasis of mutualistic core bacteria has been linked with dysbiosis and associated with pathogens (Hamdi et al. 2011; Koch and SchmidHempel 2012) and this may have happened also in the young workers in the old nests that were moved to outdoors. However, one should take care before making this conclusion not investigating variations in functions between individual bacteria species classified under the same family as was also reported by Hu et al. (2014) in the case of $C$. varians ants. In our case, the large uptake of Enterobacteriaceae does not necessarily indicate a state of dysbiosis but can also contribute to a host nutritional function, as has been reported in other insects including fruit flies and beetles (Behar et al. 2005; Vasanthakumar et al. 2006). Indeed, our BLASTn searches indicated close matches with Rahnella sp. (>99\% identity) and Y. kristensenii (100\% identity). The latter bacterial species have been isolated from wild flowers 
from a nature reserve and are associated with Apis mellifera (Vásquez et al. 2012), while the former has been isolated from the gut of the willow leaf beetle Plagiodera versicolora (Demirci et al. 2013). Rahnella aquatilis has also been isolated from the gut of other beetles such as longicorn beetles (Park et al. 2007; Podgwaite et al. 2013) and Vasanthakumar et al. (2006) described that this bacterium plays an important nitrogen-fixing role for developing larvae in the southern pine beetle Dendroctonus frontalis. Thus, we believe that the large uptake of Enterobacteriaceae can also contribute to a host nutritional function in bumblebees. However, further research is necessary before firm conclusions can be drawn. Today, there exist no specific studies dealing with the regarded non-core Enterobacteriaceae in bumblebees or other bee taxa. Since these bacteria are present in the gut of European as well as North American bumblebee species, further research is needed in this family, in relation to host functioning, next to a further interest in the functions of mutualistic core and environmental non-core bee gut microbiota.

\section{ACKNOWLEDGMENTS}

We thank Biobest for the bumblebees and Patrick Goorix for his help during the outdoors experiment. This study was supported by the Fund for Scientific Research-Flanders (Belgium).

Plasticité de la communauté bactérienne du tube digestif et présence d'Enterobacteriaceae (Gammaproteobacteria), chez le bourdon Bombus terrestris, issu de colonies élevées en laboratoire puis déplacées dans un environnement à l'extérieur

pollinisateur généraliste / microbiote / plasticité / Enterobacteriaceae / élevage de bourdons

Plastizität der Mikrobengemeinschaft des Darms und Aufnahme von Enterobacteriaceae (Gammaproteobacteria) in Hummelnestern von Bombus terrestris nach Aufzucht im Innenbereich und Verbringung nach draußen

Bombus terrestris / generalistischer Bestäuber / Mikrobiom / Plastizität / Enterobacteriaceae / Hummelzucht

\section{REFERENCES}

Anderson, K.E., Sheehan, T.H., Mott, B.M., Maes, P., Snyder, L., et al. (2013) Microbial ecology of the hive and pollination landscape: bacterial associates from floral nectar, the alimentary tract and stored food of honey bees (Apis mellifera). PLoS One 8(12), e83125

Basset, Y., Cizek, L., Cuenoud, P., Didham, R.K., Guilhaumon, F., et al. (2012) Arthropod diversity in a tropical forest. Science 338 (6113), 1481-1484

Behar, A., Yuval, B., Jurkevitch, E. (2005) Enterobacteriamediated nitrogen fixation in natural populations of the fruit fly Ceratitis capitata. Mol. Ecol. 14(9), 2637-2643

Brownlie, J.C., Johnson, K.N. (2009) Symbiont-mediated protection in insect hosts. Trends Microbiol. 17(8), 348-354

Caporaso, J.G., Lauber, C.L., Walters, W.A., Berg-Lyons, D., Lozupone, C.A., et al. (2011) Global patterns of 16S rRNA diversity at a depth of millions of sequences per sample. Proc. Natl. Acad. Sci. U. S. A. 108, 45164522

Cariveau, P.D., Powell, J.E., Koch, H., Winfree, L.T., Moran, N.A. (2014) Variation in gut microbial communities and its association with pathogen infection in wild bumble bees (Bombus ). ISME J. 8, 2369-2379

Demirci, M., Sevim, E., Demir, I., Sevim, A. (2013) Culturable bacterial microbiota of Plagiodera versicolora (L.) (Coleoptera: Chrysomelidae) and virulence of the isolated strains. Folia Microbiol. 58(3), 201-210

Dillon, R.J., Charnley, A.K. (1986) Invasion of the pathogenic fungus Metarhizium anisopliae through the guts of germ-free desert locusts, Schistocerca gregaria. Mycopathologia 96, 59-66

Dillon, R.J., Charnley, A.K. (1988) Inhibition of Metarhizium anisopliae by the gut bacterial flora of the desert locust: characterisation of antifungal toxins. Can. J. Microbiol. 34, 1075-1082

Dillon, R.J., Webster, G., Weightman, A.J., Charnley, A.K. (2010) Diversity of gut microbiota increases with aging and starvation in the desert locust. Antonie Van Leeuwenhoek 97 (1), 69-77

Edgar, R.C., Haas, B.J., Clemente, J.C., Quince, C., Knight, R. (2011) UCHIME improves sensitivity and speed of chimera detection. Bioinformatics 27 (16), 2194-2200

Engel, P., Moran, N.A. (2013) The gut microbiota of insects - diversity in structure and function. FEMS Microbiol. Rev. 37 (5), 699-735

Engel, P., Kwong, W.K., Moran, N.A. (2013) Frischella perrara gen. nov., sp nov., a gammaproteobacterium isolated from the gut of the honeybee, Apis mellifera. Int. J. Syst. Evol. Microbiol. 63, 3646-3651

Evans, J. D., Schwarz, R. S. (2011) Bees brought to their knees: microbes affecting honey bee health. Trends Microbiol. 19, 614-620

Forsgren, E., Olofsson, T. C., Vasquez, A., Fries, I. (2010) Novel lactic acid bacteria inhibiting Paenibacillus larvae in honey bee larvae. Apidologie. 41, 99-108 
Fürst, M.A., McMahon, D.P., Osborne, J.L., Paxton, R.J., Brown, M.J.F. (2014) Disease associations between honeybees and bumblebees as a threat to wild pollinators. Nature 506 (7488), 364-366

Ghazoul, J. (2005) Buzziness as usual? Questioning the global pollination crisis. Trends Ecol. Evol. 20(7), 367-373

Goulson, D. (2010) Bumblebees; their behaviour, ecology and conservation. Oxford University Press, Oxford

Hamdi, C., Balloi, A., Essanaa, J., Crotti, E., Gonella, E., et al. (2011) Gut microbiome dysbiosis and honeybee health. J. Appl. Entomol. 135(7), 524-533

Hu, Y., Lukasik, P., Moreau, C.S., Russell, J.A. (2014) Correlates of gut community composition across an ant species (Cephalotes varians) elucidate causes and consequences of symbiotic variability. Mol. Ecol. $23(6), 1284-1300$

Kaltenpoth, M. (2011) Honeybees and bumblebees share similar bacterial symbionts. Mol. Ecol. 20 (3), 439440

Killer, J., Kopecny, J., Mrazek, J., Rada, V., Benada, O., et al. (2009) Bifidobacterium bombi sp nov., from the bumblebee digestive tract. Int. J. Syst. Evol. Microbiol. 59, 2020-2024

Killer, J., Kopecny, J., Mrazek, J., Rada, V., Dubna, S., et al. (2010) Bifidobacteria in the digestive tract of bumblebees. Anaerobe 16(2), 165-170

Killer, J., Kopecny, J., Mrazek, J., Koppova, I., Havlik, J., et al. (2011) Bifidobacterium actinocoloniiforme sp nov and Bifidobacterium bohemicum sp nov., from the bumblebee digestive tract. Int. J. Syst. Evol. Microbiol. 61, 1315-1321

Killer, J., Votavova, A., Valterova, I., Vlkova, E., Rada, V., Hroncova, Z. (2014) Lactobacillus bombi sp nov., from the digestive tract of laboratory-reared bumblebee queens (Bombus terrestris). Int. J. Syst. Evol. Microbiol. 64, 2611-2617

Klungness, L.M., Peng, Y.S. (1984) A histochemical study of pollen digestion in the alimentary canal of honeybees (Apis mellifera L.). J. Insect Physiol. 30 (7), 511521

Koch, H., Schmid-Hempel, P. (2011a) Socially transmitted gut microbiota protect bumble bees against an intestinal parasite. Proc. Natl. Acad. Sci. USA. 108, 19288-19292

Koch, H., Schmid-Hempel, P. (2011b) Bacterial communities in central European bumblebees: low diversity and high specificity. Microb. Ecol. 62 (1), 121-133

Koch, H., Schmid-Hempel, P. (2012) Gut microbiota instead of host genotype drive the specificity in the interaction of a natural host-parasite system. Ecol. Lett. 15 (10), 1095-1103

Koch, H., Cisarovsky, G., Schmid-Hempel, P. (2012) Ecological effects on gut bacterial communities in wild bumblebee colonies. J. Anim. Ecol. 81 (6), 1202-1210

Koch, H., Abrol, D.P., Li, J.L., Schmid-Hempel, P. (2013) Diversity and evolutionary patterns of bacterial gut associates of corbiculate bees. Mol. Ecol. 22 (7), 2028-2044
Kwong, W. K., Moran, N. A. (2013) Cultivation and characterization of the gut symbionts of honey bees and bumble bees: description of Snodgrassella alvi gen. nov., sp nov., a member of the family Neisseriaceae of the Betaproteobacteria, and Gilliamella apicola gen. nov., sp nov., a member of Orbaceae fam. nov., Orbales ord. nov., a sister taxon to the order 'Enterobacteriales' of the Gammaproteobacteria. Int. J. Syst. Evol. Microbiol. 63, 2008-2018

Kwong, W.K., Engel, P., Koch, H., Moran, N.A. (2014) Genomics and host specialization of honey bee and bumble bee gut symbionts. Proc. Natl. Acad. Sci. U. S. A. $111(31), 11509-11514$

Lebuhn, G., Droege, S., Connor, E.F., Gemmill-Herren, B., Potts, S.G., et al. (2013) Detecting insect pollinator declines on regional and global scales. Conserv. Biol. 27 (1), 113-120

Li, L., Praet, J., Borremans, W., Olga, C., Nunes, O.C., Manaia, C.M., Cleenwerck, I., Meeus, I., Smagghe, G., De Vuyst, L., Vandamme, P.A. (2015) Bombella intestini gen. nov., sp. nov., a novel acetic acid bacterium isolated from bumble bee crop. Int. J. Syst. Evol. Microbiol. 65, 267-273

Martinson, V. G., Danforth, B. N., Minckley, R. L., Rueppell, O., Tingek, S., Moran, N. A. (2011) A simple and distinctive microbiota associated with honey bees and bumble bees. Mol. Ecol. 20, 619-628

Martinson, V.G., Moy, J., Moran, N.A. (2012) Establishment of characteristic gut bacteria during development of the honeybee worker. Appl. Environ. Microbiol. 78(8), 2830-2840

Martinson, V.G., Magoc, T., Koch, H., Salzberg, S.L., Moran, N.A. (2014) Genomic features of a bumble bee symbiont reflect its host environment. Appl. Environ. Microbiol. 80 (13), 3793-3803

McFrederick, Q. S., Cannone, J. J., Gutell, R. R., Kellner, K., Plowes, R. M., Mueller, U. G. (2013) Specificity between Lactobacilli and Hymenopteran hosts is the exception rather than the rule. Appl. Environ. Microbiol. 79, 1803-1812

Meeus, I., Brown, M.J.F., de Graaf, D.C., Smagghe, G. (2011) Effects of invasive parasites on bumble bee declines. Conserv. Biol. 25 (4), 662-671

Meeus, I., Mommaerts, V., Billiet, A., Mosallanejad, H., Van de Wiele, T., et al. (2013) Assessment of mutualism between Bombus terrestris and its microbiota by use of microcolonies. Apidologie 44 (6), 708-719

Meeus, I., Parmentier, L., Billiet, A., Maebe, K., Van Nieuwerburgh, F., et al. (2015) MiSeq(R) 16S rRNA amplicon sequencing demonstrates that indoor reared bumblebees (Bombus terrestris) harbor a core subset of bacteria normally associated with the wild host. PLoS One 10 (4), e0125152

Mohr, K. I., Tebbe, C. C. (2007) Field study results on the probability and risk of a horizontal gene transfer from transgenic herbicide-resistant oilseed rape pollen to gut bacteria of bees. Appl. Microbiol. Biotechnol. 75, $573-582$ 
Mommaerts, V., Sterk, G., Smagghe, G. (2006) Hazards and uptake of chitin synthesis inhibitors in bumblebees Bombus terrestris. Pest Manag. Sci. 62, 752-758

Moran, N.A., Hansen, A.K., Powell, J.E., Sabree, Z.L. (2012) Distinctive gut microbiota of honey bees assessed using deep sampling from Individual worker bees. PLoS One 7 (4), e36393

Newton, I.L.G., Roeselers, G. (2012) The effect of training set on the classification of honey bee gut microbiota using the Naive Bayesian Classifier. BMC Microbiol. 12, 221-230

Park, D.S., Oh, H.W., Jeong, W.J., Kim, H., Park, H.Y., et al. (2007) A culture-based study of the bacterial communities within the guts of nine longicorn beetle species and their exo-enzyme producing properties for degrading xylan and pectin. J. Microbiol. 45 (5), 394 401

Parmentier, L., Meeus, I., Cheroutre, L., Mommaerts, V., Louwye, S., et al. (2014) Commercial bumblebee hives to assess an anthropogenic environment for pollinator support: a case study in the region of Ghent (Belgium). Environ. Monit. Assess. 186(4), 2357-2367

Podgwaite, J.D., D'Amico, V., Zerillo, R.T., Schoenfeldt, H. (2013) Bacteria associated with larvae and adults of the Asian longhorned beetle (Coleoptera: Cerambycidae). J. Entomol. Sci. 48 (2), 128-138

Powell, J.E., Martinson, V.G., Urban-Mead, K., Moran, N.A. (2014) Routes of acquisition of the gut microbiota of the honey bee Apis mellifera. Appl. Environ. Microbiol. 80 (23), 7378-7387
Praet, J., Meeus, I., Cnockaert, M., Smagghe, G., Vandamme, P.A. (2015) Bifidobacterium commune sp. nov. isolated from the bumble bee gut. Antonie Van Leeuwenhoek 107 (5), 1307-1313

Salem, H., Kreutzer, E., Sudakaran, S., Kaltenpoth, M. (2012) Actinobacteria as essential symbionts in firebugs and cotton stainers (Hemiptera, Pyrrhocoridae). Environ. Microbiol. 15, 1956-1968

Schloss, P.D., Westcott, S.L., Ryabin, T., Hall, J.R., Hartmann, M., et al. (2009) Introducing Mothur: Open-source, platform-independent, communitysupported software for describing and comparing microbial communities. Appl. Environ. Microbiol. 75 (23), 7537-7541

Vasanthakumar, A., Delalibera, I., Handelsman, J., Klepzig, K.D., Schloss, P.D., et al. (2006) Characterization of gut-associated bacteria in larvae and adults of the southern pine beetle, Dendroctonus frontalis Zimmermann. Environ. Entomol. 35(6), 1710-1717

Vásquez, A., Forsgren, E., Fries, I., Paxton, R.J., Flaberg, E., et al. (2012) Symbionts as major modulators of insect health: Lactic acid bacteria and honeybees. PLoS One 7 (3), e33188

Wang, Y., Naumann, U., Wright, S.T., Warton, D.I. (2012) Mvabund- an R package for model-based analysis of multivariate abundance data. Methods Ecol. Evol. $3(3), 471-474$

Yun, J.H., Roh, S.W., Whon, T.W., Jung, M.J., Kim, M.S., et al. (2014) Insect gut bacterial diversity determined by environmental habitat, diet, developmental stage, and phylogeny of host. Appl. Environ. Microbiol. 80 (17), 5254-5264 